\title{
Về các phép mã hoá hợp lý
}

\author{
Huỳnh Hũu Nghĩa
}

\section{Một số định nghĩa}

1. Quan hệ tồng quát $\mathbf{R}$ gồm tập các thuộc tỉnh $d R^{+}$và tập các ràng buộc phụ thuộc $F$. Ta viết $R=\left\langle R^{+}, F\right\rangle$.

2. Xem hai tập $A, B \subseteq R^{+}$. Nếu $B$ phụ thuộc hàm vào $A$ ta ký hiệu $A \rightarrow B \in F^{* *}$, với $F^{* *}$ là bao đóng cùa $F$ suy tì hệ luật dần Amstrong.

3. Cho hai tập $A, B \subseteq R^{+}$: ta ghi $A \vee B$ là hội cuia hai tập $A$ và $B, A \wedge B$ là giao cua hai tập này.

4. Xem $A \subseteq R^{+}$. Tập hợp:

$$
\mathrm{Cl}_{F}(A)=\left\{b \in R^{+}: \exists A^{\prime} \in A: A^{\prime} \rightarrow b \in F^{* *}\right\}
$$

được gọi là bao đóng cuia tập $A$ ựng với tập fth (phụ thuộc hàm) $F$.

5. Cho $A, B \in R^{+}$và $A-B F^{* *}$. Ta nói $A \rightarrow B$ là một fth nguyên tố nếu và chì néu: mọi $A^{\prime} \subset A$, nếu $A \rightarrow B \in F^{* *}$ thì $A=A^{\prime}$.

6. Xem tập $K \subset R^{+}$. $K$ được gọi là chía khóa của quan hệ $R=<R^{+}, F>$ nếu $K-R^{+}$ nguyên tố trong $F^{* *}$.

7. Một fth $A-B \in F^{* *}$ được gọi là fth nội bộ của một quan hệ $\left\langle T_{i}^{+}, F_{i}>\right.$ nếu $A \vee B \subseteq T_{i}$ và $A$ là một chía khóa của $\left\langle T_{i}^{+} . F_{i}\right\rangle$.

\section{Cách mã hóa hợp lý trên tập cách biệt các thuộc tính}

Xem quan hệ tồng quát $R=<R^{+}, F>$. Ta gọi một phân hoạch (partition) $M\left(R^{+}\right)$ trên tập $R^{+}$là một cách mà hóa họ̣n lị trên quan hệ $R$ nếu $M\left(R^{+}\right)$thỏa màn các điều kiện sau: 
1. Từng vế trái và tưng vế phài cula tưng fth thuộc $F$ là hội cách biệt cula một số tập trong $M\left(R^{+}\right)$.

2. Tập các thuộc tỉnh không thuộc về phía nào của $F$ là hội cách biệt cuia một số tập trong $M(R+)$.

3. Hai tập $T_{i}, T_{j} \in M(F)$ nó́u $T_{i} \wedge T_{j} \neq 0$ thì $T_{i}=T_{j}$.

Ta dự dịnh gán cho mồi tập trong $M\left(R^{+}\right)$một con số tì 1 dến $m$ (duy nhất). Như vậy ta biến đồi tập $F$ thành tập $M(F)$ : tập “các fth với các vế gồm các con số”.

Nếu một tập hợp $A \subseteq R^{+}$, $A$ sè dược gọi la một tập mã hóa được theo phép $M$. Ta ghi $M(A)$ là tập các mà sổ của các tập con thành phấcủa $A$.

\section{Một số tính chất của một phép mã hóa hợp lý $M$}

Ta xem một cách mà hóa hợp lý $M\left(\mathrm{R}^{+}\right)=\left\{T_{1}, \ldots, T_{n}\right\}$.

(III.1) Mệnh đề:

I'ói $0 \leq m \leq n$, dăt $T^{m}$ là hội tụ cuia $m$ tập hợp nào dó thuọ̣c $M\left(R^{+}\right)$. Họ $\left\{T_{m}\right\}$ sẽ đóng kín với crác phép tính giao, hộ hị̣̂u cric tập hợp.

(III.2) Mệnh đề:

$A$ và B là hai tập thực tính mà hóa dực (theo $M$ ), $A=B$ nếu và chi nếu $M(A)$ $=M(B)$.

(III.3) Dịnh lý:

Cho <.M(R+), $M(F)>$ là hét quá mà hóa "ủa một phép mã hóa $M$ áp dụng trên quan hệ $<R+, \quad P_{>}$. Xem $A \rightarrow\{x\}$ lit mọt fth không tầm thường nào đó. Thế thì

(1) Tồn tại một tạp hợp $A_{0} \subseteq$ A, Ao mâ hóa duọc, $A_{0} \rightarrow\{x\} F^{* *}$. Ta biêu diênn đượ bằng sơ dô:

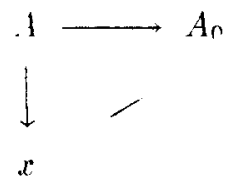

Ghi chú: Ta nói $A \rightarrow x$ là kết quả bắc cầu giữa $A \rightarrow A_{0}$ (tầm thường) và $A_{0}-x$ với $A_{0}$ mà hóa được.

(2) tồn tại một tập $B \subseteq M\left(R^{+}\right)$sao cho

- $A$ và $B$ cách biệt

- tồn tại một tập $A_{0} \subseteq A, A_{0}$ mã hóa được và $A_{0}$ xác định hàm $B$. Ta biều diexn bằng dồ thị: 


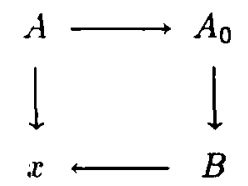

(Ta nói rằng một fth bất kỳ $A \rightarrow x$ không tầm thường có thề được xấp xỉ bằng một fth $A_{0} \rightarrow B$ với $A_{0}$ và $B$ là hai tập mã hóa được).

(III.4) Dịnh lý:

Xem tập $K \subseteq R^{+} . T$ là một tập thuợc $M\left(R^{+}\right)$.

$X e m T_{1} \subset T . T a$ có

$$
C l\left[(K D T) \vee T_{1}\right]=C l(K \backslash T) \vee T_{1}
$$

(ta thấy các tập "lơ cỗ không có giá trị gi trong phép tính bao dóng)

(III.4.1) Hệ quà

Xem tập $K \subseteq R^{+}$. Gọi $K_{1}$ là hội tất cả các tập con mã hóa được chứa trong $K$.

Đặt $K_{2}=K \backslash K_{1}$. ta có:

$$
\mathrm{Cl}_{F}(K)=\mathrm{CL}_{F}\left(K_{1}\right) \vee K_{2} .
$$

(III.5) Mệnh đề

$K$ là một tập mâa hóa được thi $C l_{r}(K)$ là một tập mã hóa được.

(III.6) Mệnh đề

A là một tập mã hóa đựọc.-Thế thì

$$
C l_{M(F)} M(A)=M\left[C l_{F}(A)\right]
$$

(một phép mã hóa hợp lý không làm thay đồi kết quả cuia phép tính bao đóng).

\section{Thực hiện các giải thuật truyển thống có dùng thêm cách mã hóa hợp lỷ}

Chúng ta thư xem một tiến trình sau:

$u, F$

$\downarrow$

11

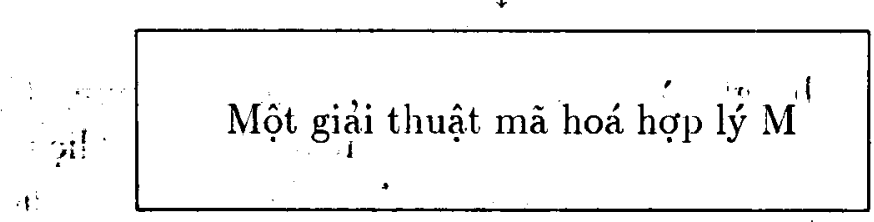




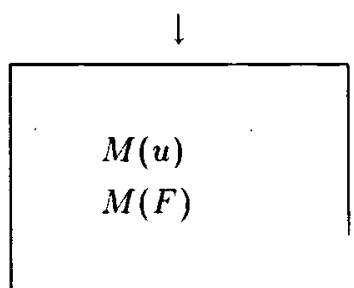

1

Một giải thuật truyển thông

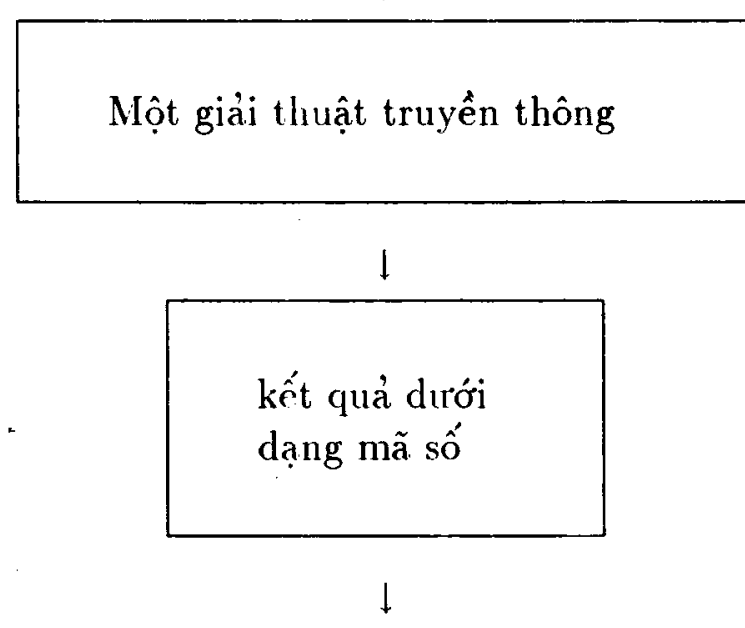

Giài thuật giải mà $M^{-1}$

$\downarrow$

Kết quả dưới dạng các thuộc tinh

Bằng các kết quả toán học trong mục (III) chúng tôi chứng minh được rằng kết quả cuối cùng không thay đồi so với cách làm thông thường là gán cho mồi thuộc tính một mã số, khi giải các bài toán truyên thống nói trên. Nói cụ thể hơn chúng tôi đã áp dụng có kết quả các giải thuật

- Tìm tất cá các chià khóa của $R$, giải thuật Lucchessi-Osborn (1978) [5].

- Giải thuật tím một cơ sờ tối thiêu [4]

- Giải thuật quan niệm lược đồ CSDL nhất quán và đầy đủ [1]

Hơn nữa khi tiến hành nghiên cưu áp dụng giải thuật [1] chúng tôi chứng minh được rằng việc biêu diễn các fth "có nguy cơ gây mâu thuân dữ liệu" sẽ được thực hiện dễ dàng và trọn vẹn nhờ các về của chưng đểu là các tập mã hóa được. 


\section{Giải thuật mã hóa hợp lý tạo ra ít mã số nhất.}

Chúng tôi xin giới thiệu một giải thuật đã được chứng minh là một phép mã hóa hợp lý và tạo ra ít mã số nhẩt (xem mục lục D.II.1 và D.II.2 trong [3]).

Trong giai đoạn mã hóa, chủng ta cần đến số thuộc tính, số phụ thuộc hàm được nhập vào và lập các sơ yếu lí lịch cuia chúng bằng các đặc tả

1) var tsốtht $=$ integer; tsốth = integer;

Dể dễ theo dõi, các cấu trúc lưu trữ chuân bị lưu trữ tối đa 24 thuộc tính và 100 phụ thuộc hàm

2) type con_trò_tht $={ }^{\wedge}$ tht;

3) var tht $=$ record $\{$ ưng tưng bước thuộc tính $\}$

tên $=$ char;

vị trí $=$ array $[1 . .200]$ of integer;

\{ thứ tự của vế, trong cấu trúc FTH, có chứa thuộc tính này \}

4) var quan_hệ $=$ array [1..24] of con_tró_tht

5) type vế $=$ set of $[\mathrm{A} . . Z]$;

tập_con = set of $[1.24]$;

6) var tập_fth $=\operatorname{array}[1 . .200]$ of vế;

mà_fth = array[1..200] of tập_con;

Cấu trúc tập_fth chừa 100 thành tố đầu đề chứa toàn các vế trái và 100 thành tố sau đề chứa toàn các vế phải. Cấu trúc mã_fth để chứa kết quả mã hóa của tập phụ thuộc hàm.

7) var CHKHOA = array [1..24] of vế

Cấu trúc CHKHOA dùng đề giải mã một tập hợp gồm các mã số thành một tập các thuộc tỉnh.

\section{Tóm lược giải thuật mã hóa NGH}

(bước 1) Sơ chế dữ liệu nhập

(1.1) - Sát nhập các fth có cùng vế trái lại đề tạo ra một fth mới có vế phải bằng hội các vế phải cũ.

- Loại bó các thuộc tính ở vế phải của một fth, đã có mặt ở vế trái. Ví dụ: biến đồi $A B C \rightarrow A D C$ thành $A B C \rightarrow D$

(1.2) - Dếm số $\mathrm{fth}$ và số thuộc tính nhập vào

- Ghi lại thuộc tính nào ờ vế nào của fth nào và nhờ vậy biết được có tất cả bao nhiêu vế chứa thuộc tính đó.

(bước 2) Sắp xếp array các con tró quan_hệ đề chuấn bị xứ lý các thuộc tính theo chiêu giam dần tông số vế có chứa thuộc tînh đó

(bước 2) Lặp đi lặp lại đến hết các thuộc tînh thuộc ít nhất một vế.

(3.1) Xem thuộc tính $\mathrm{x}$.

(3.2) Xét từng thuộc tính $\mathrm{y}$ có cùng tồng số vế với $\mathrm{x}$. 
Nếu $x$ và $y$ có mặt trong cảc vế: mang mà số là $\mathrm{i}$

(a) Dưa $x$ và $y$ vào chung một tập CHKHOA [i] nào đó và tập này

(b) Che thuộc tính y lại đề lần sau không xét nữa.

(c) Ghi mã sổ $\mathrm{i}$ vào cấu trúc của mã fth. Cụ thể là nếu $\mathrm{x}$ thuộc các vế $v_{1}, \ldots, v_{m}$ thì mã số $\mathrm{i}$ được ghi vào các thành tố thư $v_{1}, \ldots, v_{m}$ của array mã fth.

(bước 4) Goom tất cả thuộc tînh không thuộc vế nào vào tập chung CHKHOA[24]. (bước 5) Trà vè các trị mã fthn và CHKHOAח

Nhận rét

(1) Ở bước 3 ta thao tác các thuộc tỉnh theo các tuân tự trên một hàng đợi:

var QUEUE $=\operatorname{array}[1.24]$ of boolean

- chì xừ lý nhừng thành tố có giá trị 1

- những thuộc lính đà giải quyết xong (đà bị che) thì thành tố tương ứng sè có giá trị 0

(2) Độ phức tạp cula các giài lhuật trên là

$$
|I|^{2}+|U| \cdot|F|
$$

- $|U|$ là các số thuộc tính dược nhập vào

- $|F|$ là độ dài lưu trữ các fth

- $|U|^{2}$ là độ dài phức tạp (tối đa) của một thuật toán săp thứ tự (xấu nhất) được xừ dụng đến.

Chưng tôi chân thành biết ơn TS Trân Hà Nam, Công ty SCITEC thành phố HCM đà bảo trợ tác già hoàn thành bài báo này.

\section{Các tài liệu tham kháo}

1. Bích Thủy, Lương Dông Thi, Une approache de conception: une base de donnćes cohérentes et complete. Luận văn tiến si số 314 khoa KH KTXH trường đại học Gènève, 1986.

2. Ultman J.D., Principles of database systems, $2^{\text {nd }}$ edition, 1982.

3. Huỳnh Hừu Nghia, Các phép mã hóa hợp lý, tiêu luận chưa công bố, 1989.

4. Beeri C. \& Berstein P.A., Computational problems related to the design of normal forms for rlational schemas, AC.M Trans. on DB systems, v.4, n. 1, 1979.

5. Hồ Thuân \& Lề Văn Bào, Sufficient conditions for which a Relation Scheme has precisely one key, Preprint Series 11. 7, 1983, Viện KHTT và ĐK Việt Nam.

\section{Abstract}

On logical codings in process designing a relational database scheme with functional dependencies. 
In process to implement a database sclicme with "good" qualities, at the first step. we often assign to each of the set of entities a number (a code). Making note that almost the complexity of conceptual algorithms is usually, at least, proportional to number of codes of attributes, and to the length of the dependency sct, we study methods numerating subsets of a "logical" partition of the set of attributes. Such methods are so-called "logical Codings".

In this paper, we study Loyical Codings on an universal $U=\langle S, F\rangle$, in which $S$ is the set of attributes, $F$ is the set of functional dependencies. Those Logical Codings are to be proven independent to all conceptual algorithms.

And at the final, we introduce the Logical Coding NGH which has low complexity and produces a minimal set of codes. 